\title{
Bilateral Afrenal Nodules Due to Histoplasmosis in an Elderly
}

\author{
Flávio Pedreira de Freitas de Carvalho ${ }^{1}$, José Antônio Esper Curiati ${ }^{1}$, Thaís Mauad ${ }^{2}$, Milena Mendes Incerti ${ }^{2}$ and Wilson Jacob Filho ${ }^{1}$ \\ ${ }^{1}$ Geriatrics Service, Hospital das Clínicas, São Paulo University Medical School; ${ }^{2}$ Department of Pathology, São Paulo University Medical \\ School; São Paulo, SP, Brazil
}

\begin{abstract}
We report a case history of an 84-year-old elderly male patient that presented with a clinical picture suggestive of adrenal failure and bilateral adrenal nodules detected by abdominal computed tomography. A fine needle-guided biopsy was inconclusive for achieving a final diagnosis. The patient died due to septic shock and the autopsy disclosed histoplasmosis with extensive bilateral necrosis of the adrenal glands. Although the adrenal involvement in chronic disseminated histoplasmosis has been described, there have been few reports of the infection being associated with adrenal insufficiency.
\end{abstract}

Key-Words: Histoplasmosis, adrenal nodule, Addison disease, elderly, Histoplasma capsulatum.

Histoplasmosis infection is acquired with the inhalation of the spores of the Histoplasma capsulatum, a dimorphic and saprophytic fungus found mainly in contaminated soils by birds and bat excreta [1]. In Brazil, the disease is endemic [2], with especially high infection rates in the southern state of Rio Grande do Sul [3].

The clinical manifestations of the disease are protean, varying from asymptomatic disease in immunocompetent individuals to disseminated disease in infants, immunosuppressed patients or in the elderly [4]. The involvement of the adrenal glands is not uncommon in the disseminated form of the disease. Patients may present asymptomatic adrenal nodules or infrequently, with an Addisonian crisis. The final diagnosis frequently requires tissue analysis.

Here, we report the case of an 84-year-old man that presented with bilateral adrenal nodules and adrenal failure symptoms; the diagnosis of histoplasmosis was established at the autopsy only. Necrotic disease of both glands was the only site of active infection.

\section{Case Report}

The patient was an 84-year-old male, and was a retired veterinary doctor. He was admitted at the Geriatrics Ward due to asthenia and weakness that had been present for 4 months. He had been in good general health prior to the onset of symptoms of generalized weakness, decrease in food intake and progressive asthenia, which made him dependent for daily life activities. Concurrently, he developed sporadic nausea, weight loss of $15 \mathrm{~kg}$ within the period and episodic falls at home, with prodromes of dizziness and syncope. He did not refer coughing, fever, diarrhea or dyspnea.

Three months prior to the admission he was seen by a physician who started treatment for depression with paroxetine. He developed symptomatic postural hypotension, decrease in

Received on 15 October 2006; revised 21 November 2006.

Address for correspondence: Dr. Flávio Pedreira de Freitas de Carvalho. Av. Bela Cintra 450, apt 144, Consolação, São Paulo-SP, Brazil. Zip code: 01415-000. Phone: (011)32143686. E-mail: fpfcarvalho@uol.com.br.

The Brazilian Journal of Infectious Diseases 2007;11(1):160-162. (C) 2007 by The Brazilian Journal of Infectious Diseases and Contexto Publishing. All rights reserved. overall strength and periods of mental confusion with memory loss. As he developed a general and progressive worsening of the clinical picture, he was admitted at the Geriatrics Ward.

At admission, he presented mental confusion, attention deficit and disseminated muscular weakness, was dehydrated and had signs of weight loss. Arterial blood pressure (BP) was 100x65 mmHg in the sitting position and heart rate was $82 \mathrm{bpm}$. At the orthostatic position, BP was 90X60 $\mathrm{mmHg}$ and in the supine position, $\mathrm{BP}$ was $130 \mathrm{x} 80 \mathrm{mmHg}$, showing symptomatic postural hypotension. He had no changes in cardiac, pulmonary and abdominal assessment, with no palpable adenomegalies. There was no meningismus or localizing signs at the neurological assessment. The cognitive evaluation disclosed cognitive decline, through the Mini Mental State Examination: 13/30, Verbal fluency: 5 (normal value $>12$ ), and Drowning Clock Test $5 / 10$ (normal $>5$ ). Biochemical data at admission are shown in Table 1.

The abdominal ultra-sonography disclosed a chronic cholecystopathy with calculi and a distended gallbladder. The chest X-ray showed a slight heterogeneous infiltrate at the base of the left lung, with normal cardiac area. Abdominal and pelvic computed tomographies (CT) showed hypodense, solid bilateral adrenal nodules with a post-contrast increase of attenuation coefficient (Figure 1).

The cosyntropin test presented a normal pattern of response; urinary cortisol and vanylmandelic acid levels were within normal ranges. The endoscopy and colonoscopy showed no alterations; the chest CT showed signs of pulmonary congestion; the brain CT showed no abnormalities.

During the clinical evaluation the patient developed a progressive worsening of the clinical conditioning and developed pneumonia, which was treated with cephepime and oxacillin. The blood culture disclosed the presence of Staphilococus aureus sensitive to methicillin. Twelve days after the begining of antibiotic therapy, he developed oliguric acute renal failure. A peritoneal dialysis was carried out, which was complicated by abdominal wall hemorrhage that required a blood transfusion. Patient underwent hemodialysis; and a renal biopsy disclosed acute tubular necrosis. After clinical improvement, he underwent a fine-needle aspiration adrenal biopsy, which was inconclusive. A new adrenal biopsy performed a week later showed caseous necrosis with negative fast acid bacilli and fungus results. 
Table 1.

\begin{tabular}{lcc}
\hline Laboratory tests & $\begin{array}{c}\text { Values } \\
\text { at admission }\end{array}$ & $\begin{array}{c}\text { Reference } \\
\text { values }\end{array}$ \\
\hline Blood urea nitrogen & $81 \mathrm{mg} / \mathrm{dL}$ & $40 \mathrm{mg} / \mathrm{dL}$ \\
Serum creatinine & $1,8 \mathrm{mg} / \mathrm{dL}$ & $1,4 \mathrm{mg} / \mathrm{dL}$ \\
Serum potassium & $3.8 \mathrm{meq} / \mathrm{L}$ & $4 \mathrm{meq} / \mathrm{L}$ \\
Serum sodium & $134 \mathrm{meq} / \mathrm{L}$ & $140 \mathrm{meq} / \mathrm{L}$ \\
Serum albumin & $3 \mathrm{mg} / \mathrm{dL}$ & $4 \mathrm{mg} / \mathrm{dL}$ \\
Aspartate transferase & $90 \mathrm{mg} / \mathrm{dL}$ & $40 \mathrm{mg} / \mathrm{dL}$ \\
Alanine transferase & $79 \mathrm{mg} / \mathrm{dL}$ & $40 \mathrm{mg} / \mathrm{dL}$ \\
Alkaline Phosphatase & $357 \mathrm{mg} / \mathrm{dL}$ & $<250 \mathrm{mg} / \mathrm{dL}$ \\
Gama-glutamil transferase & $66 \mathrm{mg} / \mathrm{dL}$ & $<50 \mathrm{mg} / \mathrm{dL}$ \\
White Blood Cells & $\mathrm{Normal}$ & \\
Eritrocyte Sedimentation Rate & $15 \mathrm{~mm} / \mathrm{h}$ & $<10 \mathrm{~mm} / \mathrm{h}$ \\
\hline
\end{tabular}

Figure 1. Computed tomography of abdomen showing bilateral adrenal enlargment.

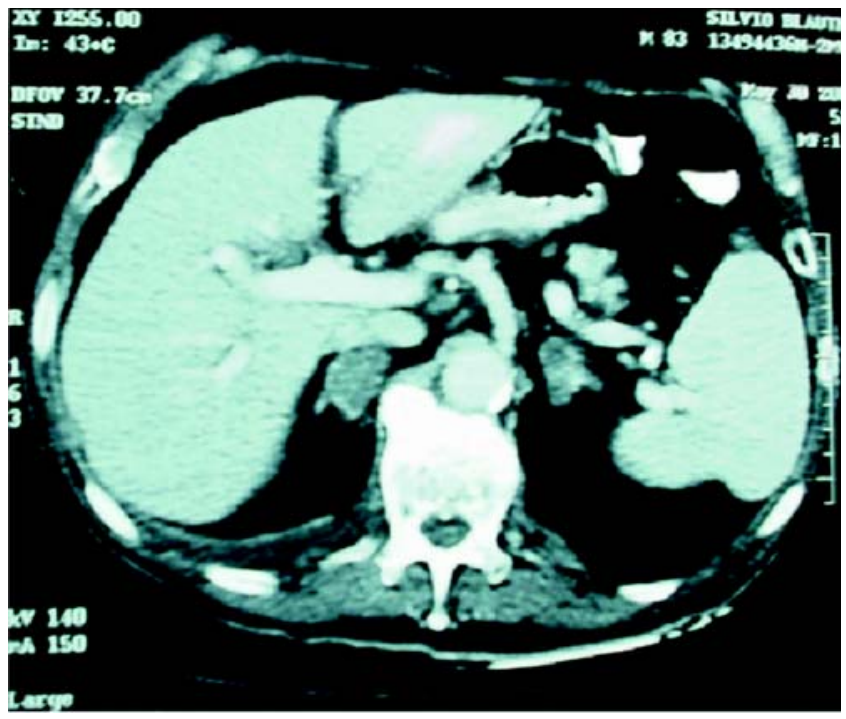

Figure 2. Adrenal gland showing extensive tissue necrosis associated with multiple minute spherical intracytoplasmatic fungic structures. H\&E, 40X.

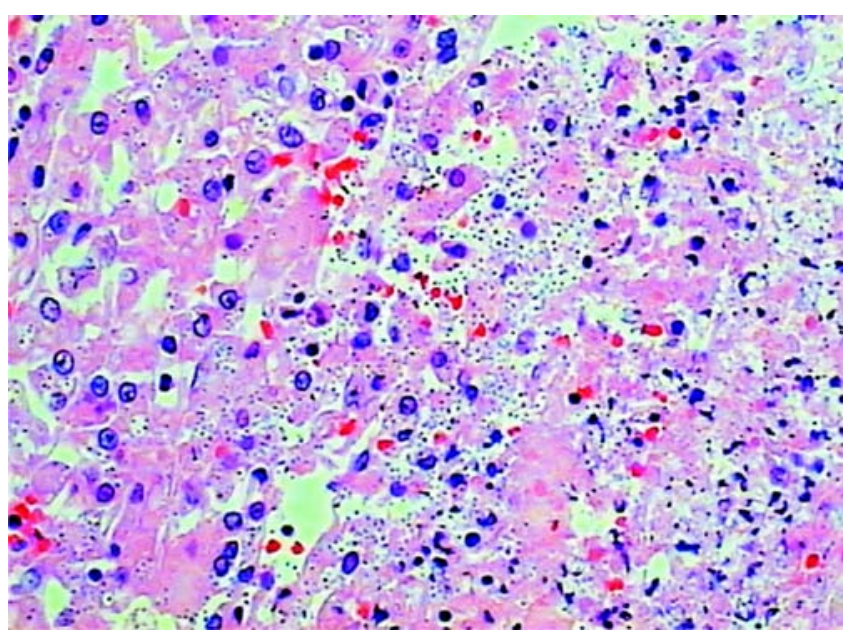

Figure 3. Positive immunostaining with the anti Histoplasma capsulatum antibody in adrenal tissue. Fungi structure stain in brown, 100X.

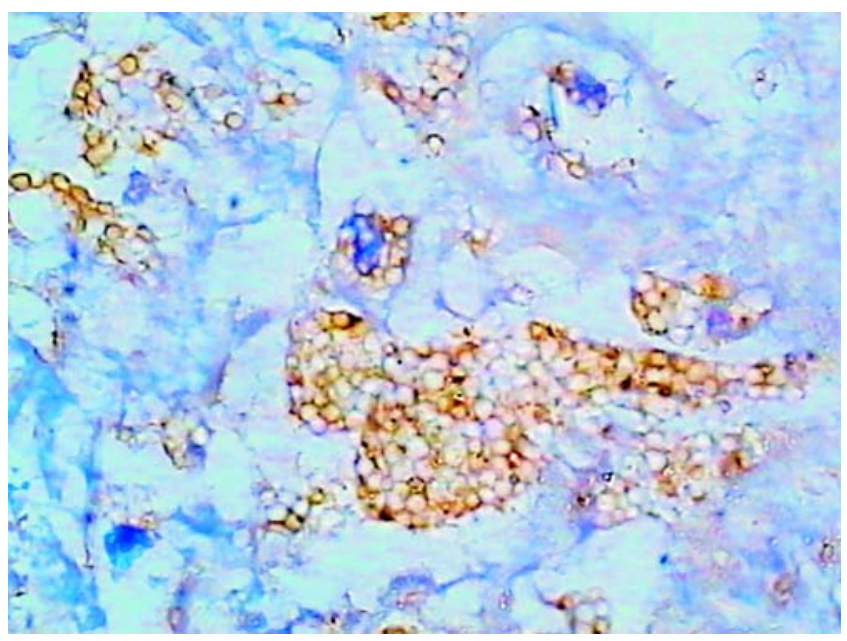

Three days after the second biopsy he developed respiratory failure, septic shock and disseminated intravascular coagulation, secondary to pneumonia by multiresistant Pseudomonas aeruginosa. He was then transferred to the Intensive Care Unit, where he died two days after.

\section{Autopsy Findings}

The autopsy showed that both adrenals were diffusely enlarged in volume, with extensive areas of parenchymal liquefying necrosis. Microscopic examination disclosed an infection by Histoplasma, with extensive parenchymal necrosis and an intense inflammatory infiltrate containing macrophages and neutrophils (Figure 2). In the macrophages (intracytoplasmatically) and within the necrotic tissue, there were several small fungal structures, Grocott stain-positive, in the shape of spores with no signs of budding (Figure 3). The immunohistochemical analysis of the sample was positive for fungal structures using an anti-Histoplasma capsulatum antibody (polyclonal antibody, Instituto de Medicina Tropical, São Paulo) and was negative with the anti-Toxoplasma gondii antibody (polyclonal antibody, DAKO, Carpinteria, USA), anti-Candida albicans (polyclonal antibody, DAKO, Carpinteria, USA) and anti-Tripanossoma cruzii antibody (polyclonal antibody, Instituto de Medicina Tropical, São Paulo). Although there was a hystiocytic reaction in the lymphoid organs, Histoplasma infection was restricted to both adrenal glands. There was a right bloody collection of approximately $500 \mathrm{~mL}$ located retroperitoneally. The lungs showed a necrotizing bacterial bronchopneumonia, which was considered the immediate cause of the patient's death.

\section{Discussion}

We report a case history of an elderly patient who presented signs of chronic hypoadrenalism and bilateral adrenal nodules, whose diagnosis of histoplasmosis was only 
established at the autopsy. While in young patients the disseminated form of the disease presents an acute and fulminating evolution, in the elderly the chronic progressive form is more frequently observed [1]. The frequency of the disseminated form is low; it is believed to be 1 in 1,000 cases [5]. Patients present with fever and unespecific symptoms, followed by pancytopenia, elevated alkaline phosphatase levels; mouth ulcers can appear. Several organs can be affected such as the lungs, gastrointestinal tract, bone marrow, central nervous system, lymph nodes and adrenal glands [1]. The dissemination occurs through the reticuloendothelial system. The diffuse involvement of the adrenals is common, but it can less frequently cause Addison's disease. This form of histoplasmosis is fatal when untreated [6].

As for the related case, although there was a clinical picture of adrenal insufficiency and -extensive necrosis of the adrenals at autopsy, the diagnosis of Addison's disease could not be established in laboratorial basis, since the cosyntropin test was within normal ranges.

The cosyntropin test has a specificity of $95 \%$ and sensitivity of $97 \%$ for primary adrenal insufficiency [7]. In several retrospective analyses using historical controls, cosyntropin tests contributed to the diagnosis of Addison disease, but these studies also showed that in some patients with Addison disease the cosyntropin test had normal values. [7].

In the elderly, histoplasmosis is usually severe and often occurs as the reactivation of a previously acquired latent infection. The case reports of histoplasmosis in the elderly show that the latency period can be quite long, varying from 10 [8] to 60 years [9]. The reactivation is commonly associated to an immunosuppressive disease, such as diabetes, but in some cases age is the only risk factor, probably due to a selective defect in the cell immunity against the histoplasm [6].

Adrenal involvement in disseminated histoplasmosis is a frequent finding, being found in up to $80 \%$ of the patients who undergo abdominal CT or ultra-sonography, or those who die due to histoplasmosis [9]. Severo et al. [3] studying patients in Rio Grande do Sul, a southern state of Brazil, and Radin [4] studying patients in the USA, observed that adrenal involvement in the disseminated form is relatively more common in HIV-negative patients than in HIV-positive ones. Moreover, Kumar et al. described 9 cases of adrenal histoplasmosis in India, all in HIV-negative patients [10].

Although the adrenal involvement in disseminated histoplasmosis has been described in several reports, hypoadrenalism has been rarely reported [11]. In elderly patients with adrenalitis due to histoplasmosis, Addison's disease seems to be also rare. In a study of elderly patients with fungal infections, Kauffman et al. found adrenal involvement in only 12 of 58 patients with histoplasmosis, and none of them showed clinical evidence of adrenal failure [6].

Radiologically, the aspects of histoplasmosis lesions seem to depend on the disease stage and the presence of liquefying necrosis. The lesions present generally as a bilateral and symmetrical enlargement with preservation of the gland shape, peripheral enhancement, central hypodensity and calcifications. However, these findings are not specific, occurring with other disseminated infections, neoplasms and subacute hemorrhage. Tissue analysis may be required for a final diagnosis. Moreover, in cases of disseminated histoplasmosis, the active infection by the histoplasm can be restricted to the adrenal gland only, being the latter the preferred site for biopsies [1].

Accordingly, in this case, the only site of active infection at the autopsy was the adrenal glands. In the present report, unfortunately, biopsy material did not yield a final diagnosis during the patient's life, probably due to the large amount of necrotic tissue present in the samples. The fungus' preference for the adrenals is not clear, but it is believed that the glucocorticoid-rich adrenal cells and the scarcity of cells from the reticuloendothelial system are contributive factors [12].

In summary, adrenal histoplasmosis may cause Addison's disease and be fatal when undiagnosed. Therefore, in endemic regions, Addison's disease due to histoplasmosis should always be included in the differential diagnosis of elderly patients with adrenal nodules.

\section{References}

1. Grover S.B., Midha N., Gupta M., et al. Imaging spectrum in disseminated histoplasmosis: case report and brief review. Australasian Radiology 2005:49,175-8.

2. Leimann B.C.Q., Pizzini C.V., Muniz M.M., et al. Histoplasmosis in a Brazilian center: clinical forms and laboratory tests. Rev Iberoam Micol 2005;22:141-6.

3. Severo L.C., Oliveira F.M., Irion K., et al. Histoplasmosis in Rio Grande do Sul, Brazil: A 21-years experience. Rev Isnt Med Trop S Paulo 2001;43.

4. Radin R. Disseminated histoplasmosis: Abdominal CT findings in 16 pacients. AJR 1991;157:955-8.

5. Umeoka S., Koyama T., Saga T., et al. High ${ }^{18} \mathrm{~F}$-fluorodeoxyglocose uptake in adrenal histoplasmosis: a case report. Eur Radiol 2005; 15:2483-6.

6. Kauffman C.A. Fungal infections in older adults. Clin Infect Dis 2001;33:550-5.

7. Dorin R.I., Qualls C.R., Crapo L.M. Diagnosis of adrenal insufficiency. Ann Intern Med 2003;139:194-204.

8. Chedid M.F., Chedid A.D., Geyer G.R., et al. Histoplasmosis presenting as addisonian crisis in an imunocompetent host. Rev Soc Bras Med Trop 2004;37:60-2.

9. Giacaglia L.R., Lin C.J., Lucon A.M., Goldman J. Disseminated histoplasmosis presenting as bilateral adrenal masses. Rev Hosp Clin Fac Med Sao Paulo 1998;53:254-6.

10. Wheat J. Histoplasmosis: experience during outbraeks Indianapolis and review of the literature. Medicine 1997;76(5):339-54.

11. Kumar N., Singh S., Govil S. Adrenal histoplasmosis: clinical presentation and imaging features in nine cases. Abdom Imaging 2003;28:703-8.

12. Rosenblit A.M., Kim A., Tuvia J., Wenig B.M. Adrenal histoplasmosis manifested as Addison's disease: unusual CT features with magnetic ressonance correlation. Clinic Radiology 2001;56(8):682-4.

13. Swartz M.A., Scolfield R.H., Dickey W.D., et al. Unilateral adrenal enlargement due to Histoplasma capsulatum. Clin Infect Dis 1996;23:813-5. 